\title{
Thermal Considerations for Designing the Next Lunar Lander
}

\author{
Matthew B. Garrison ${ }^{1 \mathrm{a}}$, Daniel H. Nguyen ${ }^{1}$ \\ IThermal Engineering Branch, NASA Goddard Space Flight Center, Greenbelt, MD 20771, USA \\ 1a(301) 286-1811, Matthew.B.Garrison@nasa.gov
}

\begin{abstract}
The Vision for Space Exploration calls for NASA to develop a lunar lander that is capable of delivering humans anywhere on the moon's surface at any time. This presents a significant challenge for thermal engineers, as the lander must be able to survive both the freezing 14-day long lunar night as well as the harsh lunar noon. These problems and potential solutions are presented for each stage of the proposed mission that will return American astronauts to the moon.
\end{abstract}

Keywords: Exploration, thermal, lander, lunar

PACSE from this list: http://www.aip.org/pacs/index.html

\section{INTRODUCTION}

The President's Vision for Space Exploration calls for NASA to return astronauts to the moon by 2020 and to establish an outpost by 2022. The goal of the lunar exploration is to learn how to live in a harsh environment for an extended period of time, and then to safely return them to Earth as a prelude to a Mars exploration program. To accomplish this mission, NASA will develop a single lunar lander capable of: (1) a 7-day sortie mission to any location on the surface at any time, (2) an extended mission to an outpost at the lunar poles. This presents a major challenge for thermal engineers, as the surface can vary from extremely hot at the sub-solar point to extremely cold at night.

The proposed mission is divided into a series of phases, as shown in Figure 1. A heavy-lift launch vehicle would place the Earth Departure Stage (EDS) and lunar lander into low-Earth orbit, where it will loiter for up to 90 days. A second launch will orbit the Crew Exploration Vehicle (CEV), which is comprised of the Crew Module (CM) and Service Module (SM), that will rendezvous with the EDS and lander. The EDS will provide the boost for the 3-day trans-lunar trajectory before detatching. The CEV will remain in lunar orbit while the lander descends to the surface for a duration of either 7 or 180 days. When the surface stay is completed, the ascent stage of the lunar lander will rendezvous with the CEV, then will detach after crew and cargo have been transferred. The CM and SM remain coupled for the return trans-lunar cruise, then detach so that the CM can perform atmospheric reentry. 


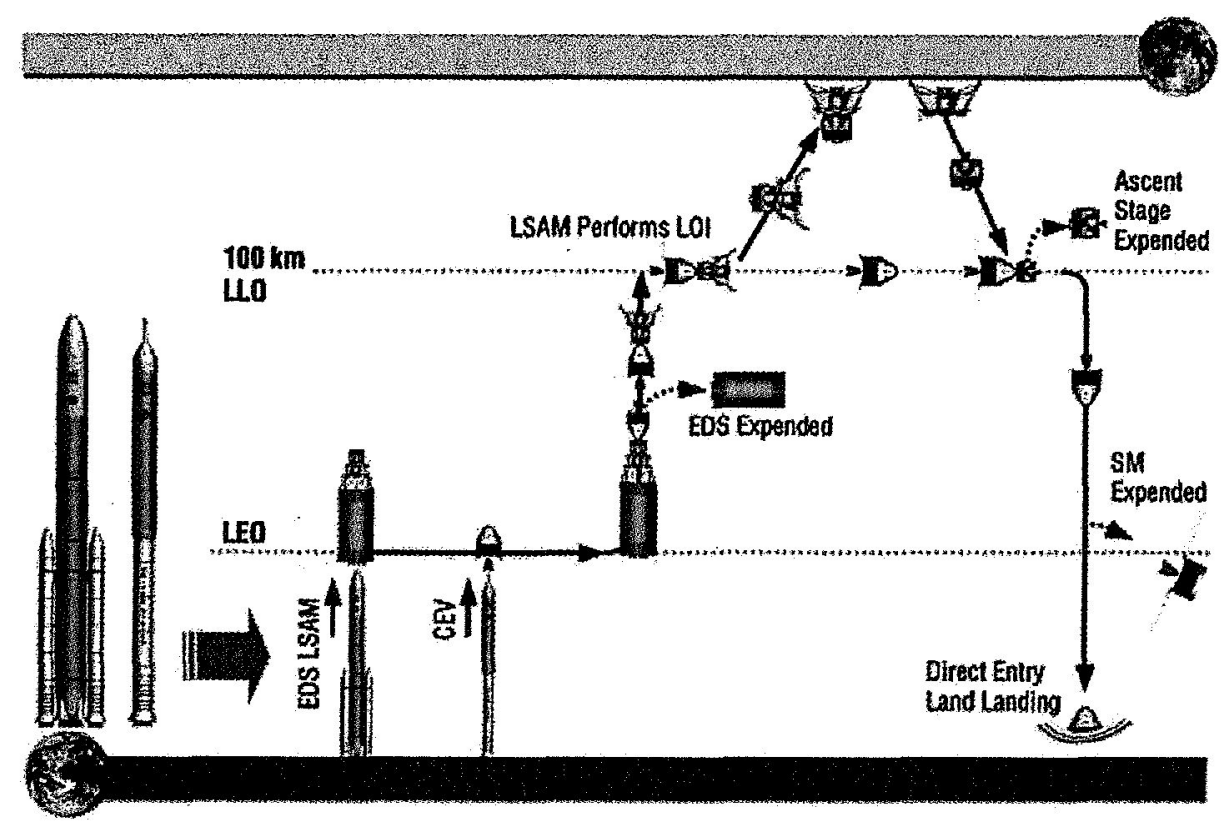

FIGURE 1. The Phases of the Missions that Will Return Astronauts to the Moon.

\section{MISSION PHASES}

The mission phases that are of the most significant to the thermal design of the lander are the Earth-orbit loiter, the trans-lunar cruise, sortie surface duration at the sub-solar point and lunar night, ascent, and the potential long-duration stay at the lunar poles to support an outpost. Thermal considerations will be discussed for each of these phases below.

\section{Earth-Orbit Loiter}

The current mission profile calls for the lunar lander and Earth Departure Stage to loiter up to 90 days in lowEarth orbit. This means that the lander will have to survive an extended time as an Earth-orbiting satellite, with a minimum mass of dedicated loiter thermal control hardware and heater power. If cryogenic fuels are used on the lander, the thermal control system will have to minimize boil-off during this period. Heat leaks will have to be compensated by launching extra cryogens. The cost is $36.5 \mathrm{~kg} / \mathrm{W}$ of $1 \mathrm{O}_{2}$ and $17.5 \mathrm{~kg} / \mathrm{W} \mathrm{of} 1 \mathrm{H}_{2}$ per unit of heat leak, plus extra mass for tanks and infrastructure.

\section{Trans-Lunar Cruise}

Over the duration of the trans-lunar cruise, the lander will remain docked with the Crew Exploration Vehicle, and therefore any roof radiators (discussed in the next phase) will lose efficiency because they will bave a blocked view to space. As Figure 2 shows, this is a function of the radiator's radial distance to the docking point, which will be along the center of mass to prevent a bending moment through the connection during thrusting. In the case of a circular design, a $5 \mathrm{~kW}$ radiator would only be capable of rejecting $3.1 \mathrm{~kW}$, although radiators will most likely be sized for the hot lunar sub-solar point. 


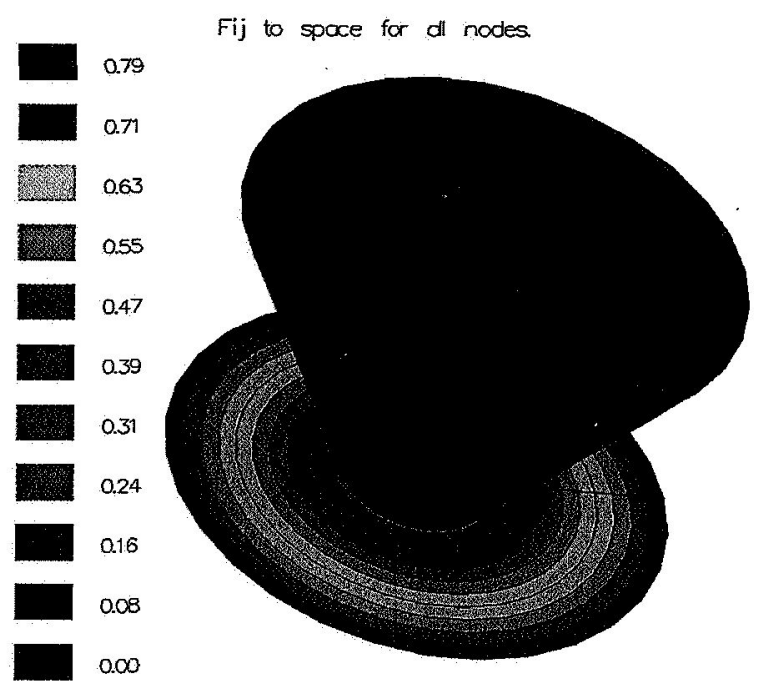

FIGURE 2. Reduced Roof Radiator View to Space while Docked to the CEV.

\section{Lunar Surface Sortie: The Sub-Solar Point}

The lunar surface presents a very harsh thermal environment. The lack of an atmosphere, coupled with low surface conductivity and high emissivity make for a variation in temperatures, ranging from $100 \mathrm{~K}$ to $380 \mathrm{~K}$. Each lunar rotation takes 28 Earth days, making it impractical to rely on thermal storage media. Additionally, the lunar regolith is composed of a statically-charged powder with high emissivity and absorptivity, which could greatly degrade the performance of any thermal system unless properly accounted for.

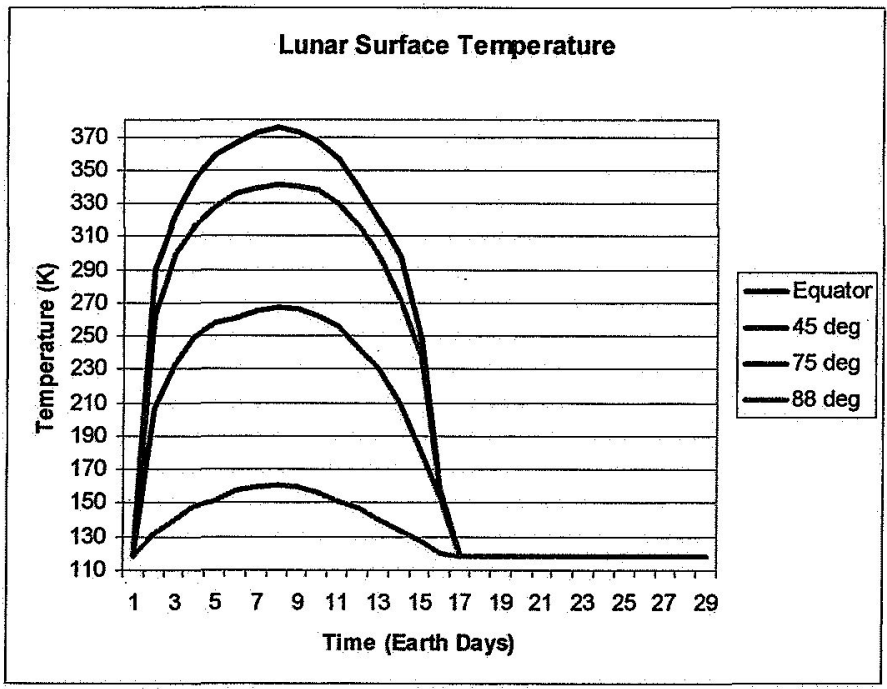

FIGURE 3: Surface Temperatures Seen at Different Lunar Latitudes through a 28-Day Lunar Rotation (Eckart, 1999).

The "Anytime, anywhere" requirement means that any lunar system must be capable of surviving on the subsolar point of the lunar surface. The solar load at this point is $1414 \pm 7 \mathrm{~W} / \mathrm{m}^{2}$ normal to the surface. Because the moon is a diffuse high-emissivity body, this results in surface IR load of $1314 \mathrm{~W} / \mathrm{m}^{2}$ at perihelion (Gilmore, 2002). 
A sunshield was proposed to block the larger of the two heat sources, but this design results in large complications. In order to test this concept, a thin-film sunshield was modeled to cover a representative cube as well as some of the surrounding regolith. The upward-facing surface was modeled with a white paint finish $(\alpha / \varepsilon$ $=0.2 / 0.92)$ to allow heat rejection, and the underside was VDA $(\alpha / \varepsilon=0.08 / 0.04)$ to minimize the heat radiated down to the surface. Analysis shows that the sunshield heats to approximately $350 \mathrm{~K}$. By blocking the view to space, this warms the radiative sink for the lander and the surface, as shown in Figure 4 . The $1414 \mathrm{~W} / \mathrm{m}^{2}$ of solar flux is thus converted to a smaller flux of $\mathbb{R}$ energy, which is at the same wavelength that the radiators are trying to reject heat. This would make it very difficult to cool the lander.

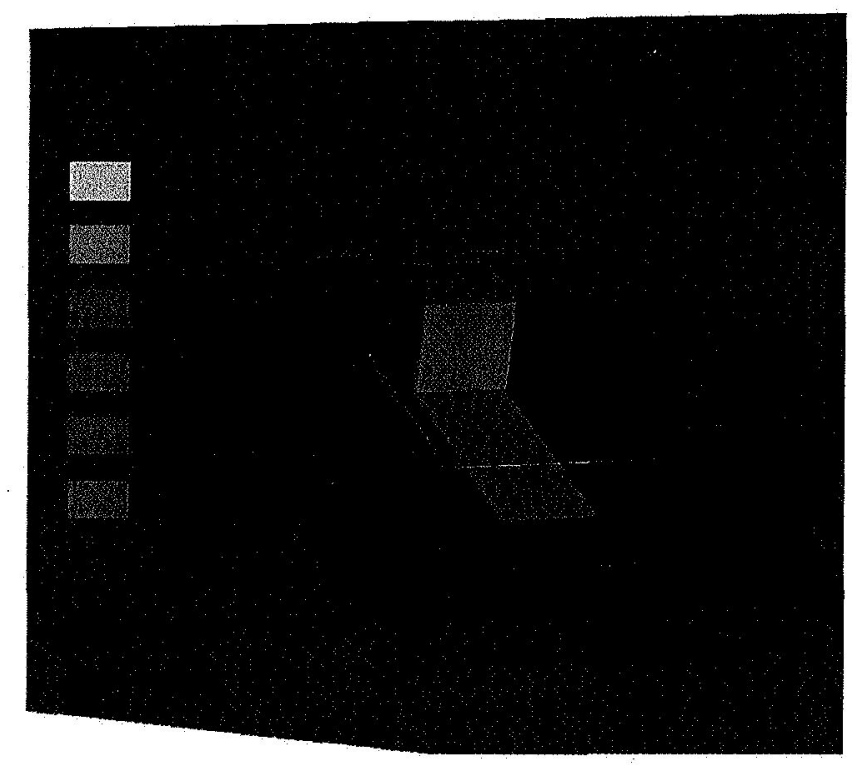

FIGURE 4. Temperature Map of a Representative Volume Covered by a Sunshield at the Lunar Sub-Solar Point.

Two methods of heat rejection were considered for the hot case: radiators mounted on the roof and heat sinks buried into the regolith. Lunar regolith has a thermal conductivity (Silk, XXX) of approximately $0.8 \mathrm{~W} / \mathrm{m} / \mathrm{K}$; modeling shows that a single heat sink held at $290 \mathrm{~K}$ would only be able to sink $15 \mathrm{~W}$. Figure 5 shows that the sink only heats the regolith in an area approximately $50 \mathrm{~cm}$ wide. 


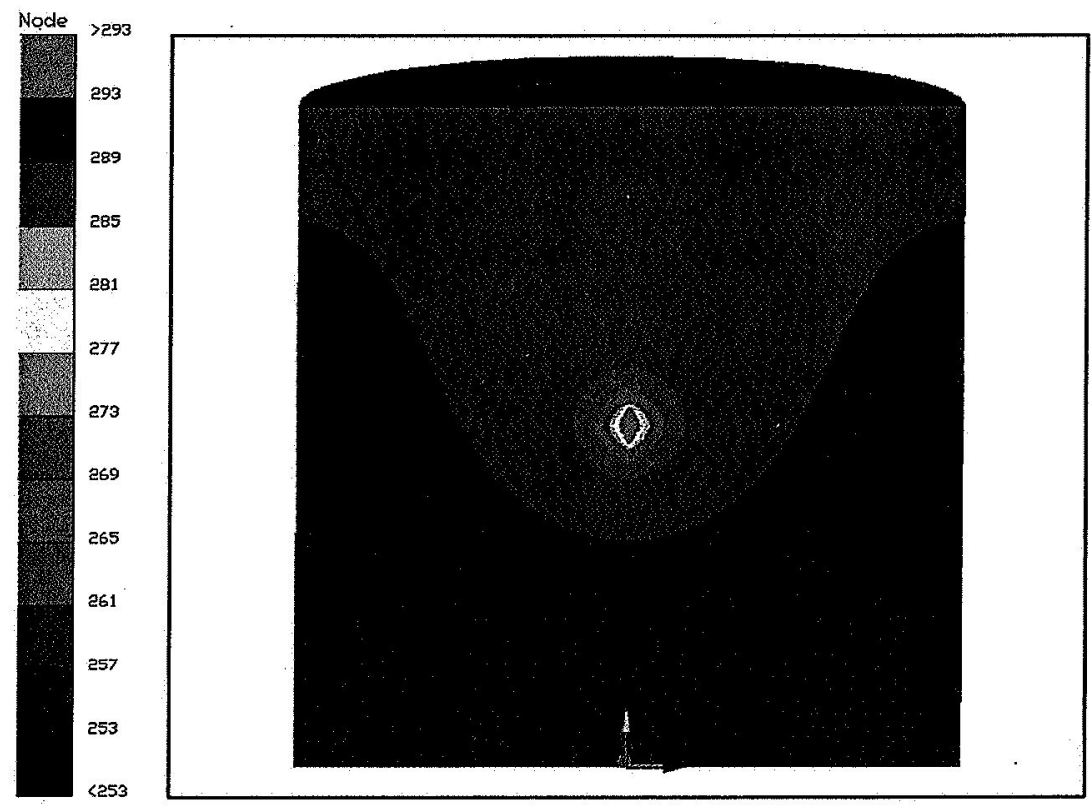

Temperature $[\mathrm{K}]$. Time $=0$ sec

FIGURE 5. Temperature Map of a 3m Diameter by 3m Height Cylinder of Regolith with a 15 W Heat Sink.

Radiators were evaluated to reject heat to space. The thermal loads discussed above require that the radiator have a low-absorptivity finish and that it is situated parallel to the surface. Two surface finishes were investigated: white paint $(\alpha / \varepsilon=0.2 / 0.92)$ and OSRs $(\alpha / \varepsilon=0.05 / 0.8)$. In addition, directional baffles were considered to limit the emission to an $80^{\circ}$ angle. This allows for $2^{\circ}$ exclusion to account for the $1.5^{\circ}$ solar tropics and $8^{\circ}$ of variation in surface profiles. This results in an area reduction factor of 0.48 . Table 1 shows the radiator effectiveness for all four combinations of these options. All other discussion of radiators in this paper assume the quartz mirror OSR surface without baffles. Consideration must also be given for the affect of lunar regolith accumulating on the radiator surface, as this will greatly increase the amount of solar load absorbed by the surface.

TABLE 1. Radiator Effectiveness for Combinations of Surface Treatments and Baffles

\begin{tabular}{lcc}
\hline Radiator Surface & Directional Baffles & Effectiveness $\left(\mathbf{m}^{2} / \mathbf{k W}\right)$ \\
\hline White Paint & No & 13.33 \\
White Paint & Yes & 8.01 \\
Optical Solar Reflector & No & 4.31 \\
Optical Solar Reflector & Yes & 9.68 \\
\hline
\end{tabular}

Any angle that allows the radiator to view the surface will overwhelm its heat rejection capability, since the surface is emitting $1314 \mathrm{~W} / \mathrm{m}^{2}$ at the same wavelengths that the radiator is emitting. Figure 6 shows how the IR load grows substantially faster than the solar load drops as the radiator is tilted off of the horizontal to reduce the incoming solar load. In addition, a small angular tilt could prevent heat pipes embedded in a large radiator from functioning in lunar gravity. 


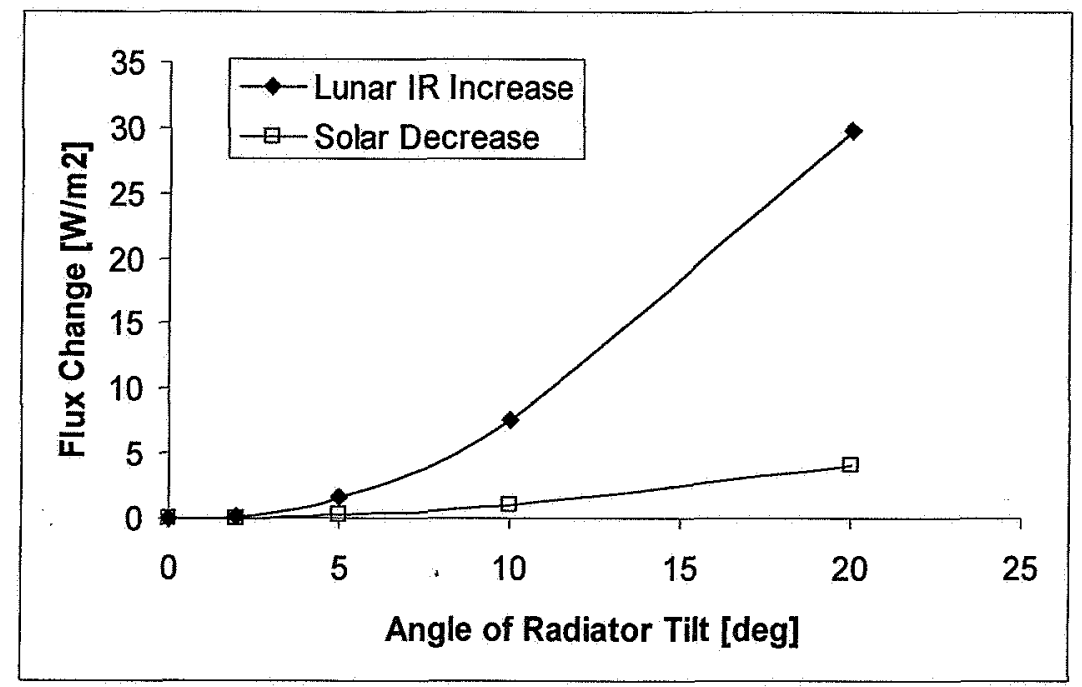

FIGURE 6. Ambient Load Change as the Radiator is Tilted Off the Horizontal Orientation.

\section{Lunar Surface Sortie: Lunar Night}

Any equipment that lands on the sunset side of the moon will likely experience at least a portion of the 14-day long lunar night. The surface in this environment is approximately $100 \mathrm{~K}$, emitting just $5.2 \mathrm{~W} / \mathrm{m}^{2}$. This presents a problem because the radiators that were designed to hold the maximum load at the sub-solar point are now oversized. As Figure 7 shows, the radiator capacity rises from $245 \mathrm{~W} / \mathrm{m}^{2}$ to $312 \mathrm{~W} / \mathrm{m}^{2}$ as it rotates from the subsolar point to the dark side, where the ambient loads disappear.

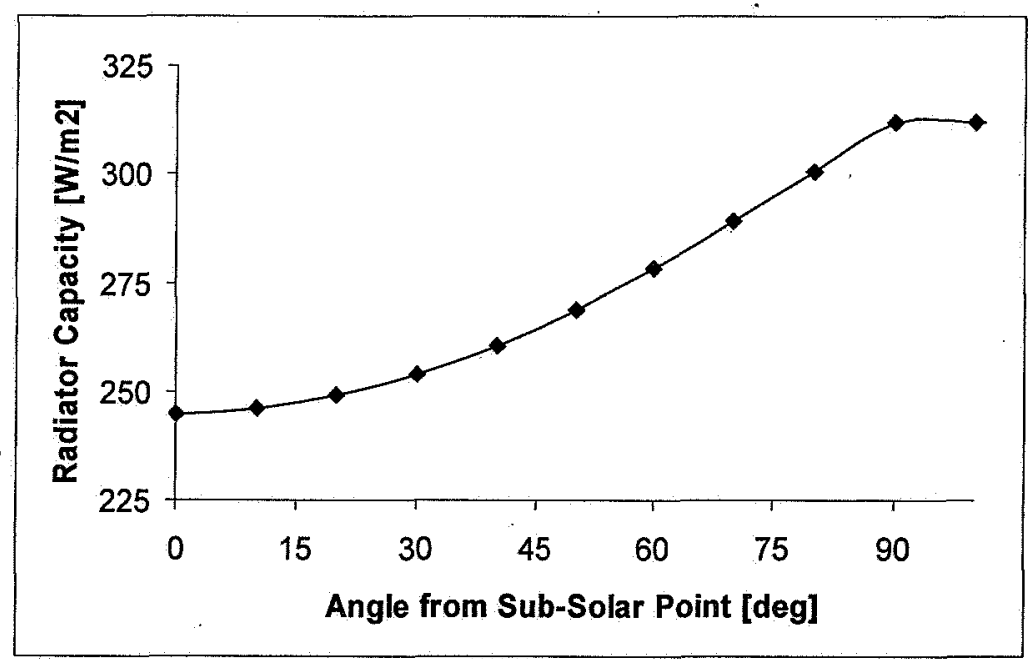

FIGURE 7. Variations in Radiator Capacity as the Moon Rotates Away From the Sub-Solar Point

In order to prevent the radiators from over-cooling the system, three options were considered. The radiator temperature could be maintained by increasing the power dissipation by $27 \%$, but this would not be feasible since power will be scarce on the unlit side of the moon. Likewise, the radiator's interchange factor with space could be reduced by $21 \%$. This could be accomplished by articulating mechanisms to cover the radiator with insulation or through a variable emittance coating, like the system employed on the Space Technology 5 mission. The simplest fix would perhaps be to disconnect $21 \%$ of the radiator from the thermal bus through heat 
switches or valves on a pumped loop. Heat would have to be applied to the radiator to maintain its temperature above the freezing point of the heat pipe fluid as shown in Figure 8.

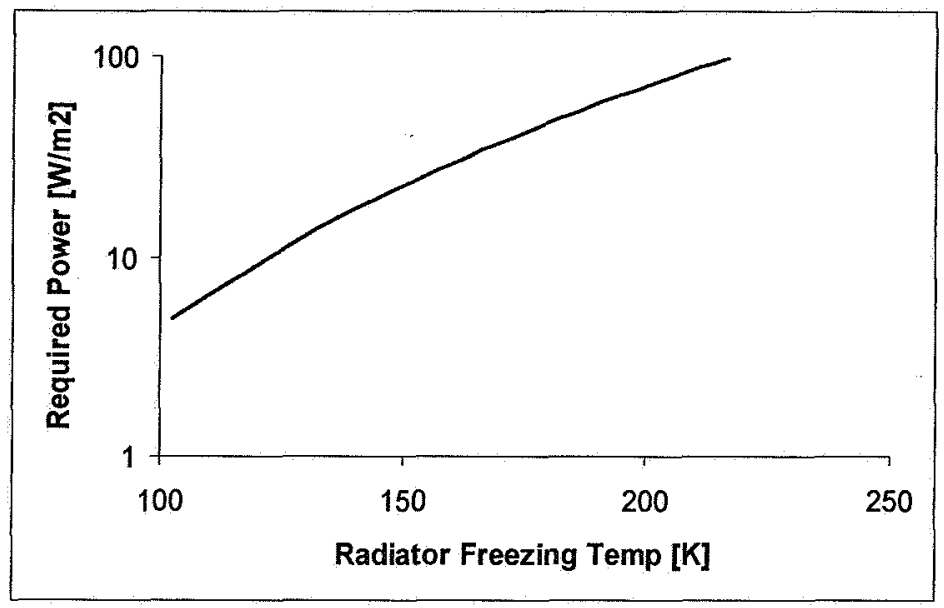

FIGURE 8. Heat Required per Unit Area to Keep an Inactive Radiator Above its Freezing Point During Lunar Night

\begin{abstract}
Ascent
A lunar lander could consist of a dedicated minimum-mass ascent stage that remains attached to the habitat until the short ascent flight. In this case the ascent stage would only be required to dissipate its own heat for the 3hour flight from the lunar surface to the CEV in lunar orbit. Due to the short time-span, phase-change systems like evaporative cooling are possible. A system would require $4.8 \mathrm{~kg} / \mathrm{kW}$ of water to power such a system for that flight time.

Considering the short activation time, an ascent stage could, instead, be designed to absorb the heat load rather than to reject it. If the heat could be evenly spread over the structure, the total temperature rise would be 12 $\mathrm{K}^{*} \mathrm{mT} / \mathrm{kW}$ assuming the majority of the stage is made of aluminum.
\end{abstract}

\title{
PHASE-INDEPENDENT CONSIDERATIONS
}

Many thermal design considerations cross over multiple mission phases. These can include the changes in requirements associated with servicing a lunar outpost, human spaceflight considerations and micrometeoroid orbital debris (MMOD) mitigation.

\section{Servicing a Lunar Outpost}

A 180-day lunar outpost provides multiple design advantages over a sortie mission. The outpost will most likely be located at a more benign environment such as the lunar poles. There it will see a near-constant environment where it will receive a low surface $\mathbb{R}$ load and steady near-horizontal solar loads. This will allow all the radiators to be sized at their maximum capacity of $312 \mathrm{~W} / \mathrm{m}^{2}$ and eliminates the need to keep the radiator from viewing the lunar surface. The extra mass available from the more-efficient radiators could be used to carry valuable logistics to the surface for the operation of the outpost.

It will be possible to do more extensive work on the outpost thermal system, whereas it would take too much time out of a short sortie schedule. This could include burying the outpost in regolith for thermal insulation as well as for radiation protection. Building up regolith embankments along the sides of the outpost would protect it from the horizontal solar flux while still leaving the top exposed for ingress/egress or communications. 
An outpost not situated at a pole will experience a cycle with 14 days of daylight and 14 days of night. The changing environment could be partially mitigated by using heat storage materials. Unfortunately, the long period of rotation drives these systems to being very large. If water was brought from Earth to store energy in the solid-liquid phase change, it would require $3.6 \mathrm{mT} / \mathrm{kW}$ of station power. Work is being done at GSFC to measure the heat capacitance of lunar regolith with the possibility that in situ resources may be capable of acting as a heat storage medium.

\section{MMOD Mitigation}

Depending on the orientation of its components, the thermal control system may be called on to either survive or provide protection from the micrometeoroid environment. Heat pipes must be laid out such that a punctured pipe will not result in a failed system, especially if it is located in a roof radiator. Furthermore, a roof radiator may be called on to mitigate impact damage to the habitat or lander. Other work (Vanzani) has shown that the average micrometeoroid is $1.5 \times 10^{-5}$ grams and is traveling at $13.3 \mathrm{~km} / \mathrm{s}$, yielding an energy of 1.33 Joules. Figure 9 shows the flux of particles to the lunar surface over an average year. The moon accretes $1.8 \times 10^{6} \mathrm{~kg}$ of mass per year, or $4.8 \times 10^{-8} \mathrm{~kg}$ per square meter of horizontal area. Assuming the average particle size and a 20 $\mathrm{m}^{2}$ horizontal radiator, a sortie mission could expect 1.2 impacts on its radiator and an outpost mission could expect 31.3 impacts.

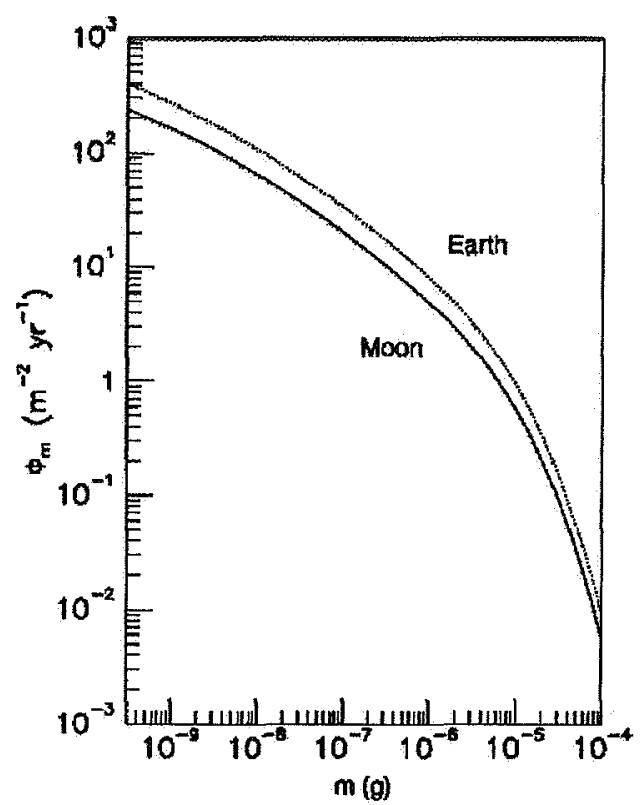

FIGURE 9. Predicted Micrometeoroid Flux to the Lunar Surface (Vanzani, 1997)

\section{Human Spaceflight}

The human element of a lunar mission presents additional requirements for a thermal design. First and foremost, they dictate that the cabin be maintained at a comfortable temperature so that the astronauts can efficiently perform their duties. In the event of a leaking pipe, heat transport fluids must be non-toxic, like an aqueous propylene glycol solution (Trevino, 2004). This has the advantage over water of having a lower freezing point and a smaller growth during freezing, reducing the requirements on external fluid lines.

\section{CONCLUSION}


The thermal design of a versatile lunar lander for the return to the moon will be a difficult multi-staged process. Unlike the Apollo-era LEM, this vehicle must be able to house astronauts in both the $400 \mathrm{~K}$ lunar sub-solar point and the $100 \mathrm{~K}$ lunar night and must be able to remain on the surface for a half-year while servicing an outpost. It must be able to meet a widely-different set of requirements for multiple mission phases with the minimum mass and power requirements on the system. Therefore, it will be necessary to consider and to implement the aforementioned points in order to have a successful lunar lander design.

\section{ACKNOWLEDGMENTS}

We thank blah blah and blah-ditty-blah-blah for all their support.

\section{REFERENCES}

Eckart, P., Lunar Base Handbook, McGraw-Hill Publishing, New York, New York, 1999, Ch. 5.

Gilmore, D., Spacecraft Thermal Control Handbook, Volume I: Fundamental Technologies, Aerospace Press, El Segundo, California, 2002, pp. 53-56.

Trevino, L., Copeland, R., Elliott, J., Weislogel, M., "Freeze Tolerant Radiator for Advanced EMU," in International Conference on Environmental Systems, SAE International, 2004, pp. 1-5.

Vanzani, V., et al., "Micrometeoroid Impacts on the Lunar Surface," Lunar and Planetary Science, 28, 1997.

Westheimer, D. T., Tuan, G. C., "Active Thermal Control System Considerations for the Next Generation of Human Rated Space Vehicles," in $43^{\text {rd }}$ ALAA Aerospace Sciences Meeting and Exhibit, Reno, Nevada, 2005, pp.1-5. 